patients. $\mathrm{CD}^{+} \mathrm{T}$ cells, derived from PBMCs of healthy donors, were used to obtain $\mathrm{T}$-cell clones. $\mathrm{CD}^{+}{ }^{+} \mathrm{T}$-cell clones were classified on the basis of their ability to produce IFN- $\gamma$ and/or IL-17. T cell clones were polyclonally stimulated with anti-CD3/CD28. Cytokine expression was assessed with RT-PCR and production in supernatants by ELISA.

Results As previously described, IL-17A and TNF- $\alpha$ had a synergistic effect for both cells. Moreover, a stronger induction for IL-6 production was seen for synoviocytes compared to EC $(1005 \pm 22 \mathrm{pg} / \mathrm{ml}$ versus $250 \pm 15 \mathrm{ng} / \mathrm{ml}$ respectively). Supernatants from inactivated Th1 or Th17 clones had no effect on EC. Regarding synoviocytes, supernatants from inactivated clones induced IL- 6 and IL- 8 mRNA with a stronger effect for Th17 cells (1000 fold versus 100 fold compared to resting synoviocytes, $p=0.045)$. While activated Th1 supernatants had a strong effect on EC and increased the expression of IL-6, IL-8, E-selectin, and tissue factor mRNA (98.12, 89 fold respectively, compare to control), Th17 supernatants had no effect. Activated Th1 and Th17 supernatants had the same effect on synoviocytes (254 and 754 fold compared to control). Th1 cells were more potent inducers of IL-6, IL-8 and tissue factor mRNA in EC than Th17 which had no significant effect $(5,12.5$, 7.5 fold respectively compared to control). Th17 cells were most effective to stimulate IL- 6 and IL-8 mRNA expression in synoviocytes compared to Th1 (100 fold versus 24 for IL-6 mRNA, $p=0.027$ ). Anti-IL-17 antibody reduced IL-6 production from $20.0 \pm 4.5 \mathrm{ng} / \mathrm{mL}$ to $13.2 \pm 3.9$ $\mathrm{ng} / \mathrm{ml}(\mathrm{p}=0.032)$ in synoviocytes cultured with Th17 clones.

Conclusions While Th1 cells were able to induce inflammatory cytokine expression in EC and synoviocytes, Th17 cells were only active to promote inflammation in inflammatory cells such as synoviocytes. The effect of Th17 cells appears to depend of IL-17 and the type of stromal cells.

\section{A3.8 EXTRATHYMIC AUTOIMMUNE REGULATOR (AIRE) EXPRESSION IN RHEUMATOID ARTHRITIS}

doi:10.1136/annrheumdis-2013-203216.8

AR Noort, KPM van Zoest, MC Lebre, PP Tak, SW Tas. Division of Clinical Immunology \& Rheumatology, Academic Medical Center, University of Amsterdam, The Netherlands

Background AIRE is a transcription factor that is involved in the negative selection of self-reactive thymocytes in the thymus. Recently, AIRE protein has also been detected in peripheral lymphoid organs, predominantly in dendritic cells (DC). In these peripheral sites, AIRE was found to regulate the expression of a group of tissue-specific antigens that is distinct from those expressed in the thymus, suggesting that peripheral AIRE may play a complementary role in tolerance induction. It is currently unknown whether AIRE may play a role in inflamed tissues, such as rheumatoid arthritis (RA) synovial tissue (ST).

Objective To document and further characterise extrathymic AIRE expressing cells in ST and paired peripheral blood (PB) mononuclear cells (MCs) as well synovial fluid (SF) MCs of RA patients. Methods ST was obtained via mini-arthroscopy from inflamed joints of RA or undifferentiated arthritis (UA) patients. Expression of AIRE was evaluated using IHC and IF microscopy. AIRE expression was also investigated in PB and SF DC using flow cytometry. Results AIRE expressing cells were detected in $80 \%$ of analysed RA ST and in contrast only in $25 \%$ of UA ST. Further characterisation using double-immunofluorescence microscopy revealed that these cells were predominantly CD1c+ myeloid (m)DC. Interestingly, a significantly higher percentage of $\mathrm{CD} 1 \mathrm{c}+\mathrm{mDC}$ in RA SF expressed AIRE $(55 \pm 5 \%)$ compared to RA PB $(20 \pm 3 \%)$ and healthy PB $(19.7 \pm 2 \%)$

Conclusions Extrathymic AIRE expressing $\mathrm{CD} 1 \mathrm{c}+\mathrm{mDC}$ are present in RA ST and RA SF, suggesting a role in synovial inflammation. Extrathymic AIRE expression in RA synovial inflammation may be an attempt to control inflammation through the induction of peripheral tolerance to antigens involved in the perpetuation of the chronic inflammatory response.

\section{A3.9 IL-17RA SIGNALLING IS ESSENTIAL FOR COLLAGEN INDUCED ARTHRITIS DEVELOPMENT}

doi:10.1136/annrheumdis-2013-203216.9

1.20dilia BJ Corneth, 1,2Adriana MC Mus, 1,2Patrick S Asmawidjaja, 1,2Maarten D Brem, ${ }^{1,2}$ Franka Luk, ${ }^{1,2}$ Wendy Dankers, ${ }^{3}$ Rudi W Hendriks, ${ }^{1,2}$ Erik Lubberts. ${ }^{1}$ Departments of Rheumatology; ${ }^{2}$ mmunology; ${ }^{3}$ Pulmonary Medicine, Erasmus MC, University Medical Center, Rotterdam, The Netherlands

Introduction IL-17A plays an important role in collagen-induced arthritis (CIA). It signals through the IL-17 receptor (IL-17R) A and $\mathrm{C}$ heterodimer. The IL-17RA appears to be a common receptor subunit for several IL-17 cytokine family members, including IL-17A, IL-17C and IL-17F. Lack of IL-17RA signalling may therefore have a broader effect than lack of IL-17A alone. We therefore aim to determine the role of IL-17RA signalling in arthritis.

Methods Disease incidence and severity were scored in wild type (positive control), IL-17RA deficient and IL-23p19 deficient (negative control) mice in CIA. T helper cell profiles and humoral immune responses were analysed by flow cytometry and immuno-histochemistry. Serum auto-reactive IgG antibodies were measured by ELISA. Pathogenicity of $\mathrm{T}$ cells and total splenocytes was determined in a functional assay in vitro.

Results As expected, wild type mice developed CIA from day 21 after the first immunisation. IL-23p19 deficient mice did not develop arthritis. Interestingly, IL-17RA deficient mice were completely protected against CIA, even after a third immunisation. This is in contrast to IL-17A deficient mice, of which $20 \%$ is still susceptible to CIA. T cells in IL-17RA deficient mice display a Th2-like phenotype in CIA with higher proportions of IL-4 producing CD4 T cells. This population is distinct from IL-17A producing $\mathrm{T}$ cells. The shift in T cell phenotype induces a less inflammatory B cell response with fewer plasma cells in the spleen and lower pathogenic IgG2a antibody production in favour of IgG1 production. In a functional assay, both isolated CD4+ T cells and total splenocytes of IL-17RA deficient mice were less capable of inducing pro-inflammatory IL-6 production by normal, IL-17RA expressing synovial fibroblasts in CIA in vitro.

Conclusions Here we show that lack of IL-17RA signalling prevents auto-immune inflammation of the joint. In addition, $T$ helper cells shift to a Th2 like phenotype characterised by IL-4 production. $\mathrm{T}$ cells and splenocytes of these mice are less pathogenic, leading to lower pathogenic IgG2a antibody levels in serum. This is in contrast with IL-17A deficient mice and suggests that other factors involved in CD4 + T cell differentiation and pathogenicity can signal through the IL-17RA.

\section{A3.10 IL-6 RECEPTOR BLOCKADE ENHANCES CD39+ REGULATORY T-CELL DEVELOPMENT IN RHEUMATOID ARTHRITIS AND IN EXPERIMENTAL ARTHRITIS}

doi:10.1136/annrheumdis-2013-203216.10

'Allan Thiolat, 'Jerome Biton, 'Luca Semerano, ${ }^{2} Y$ ves-Marie Pers, ${ }^{2}$ Pierre Portales, ${ }^{1}$ Delphine Lemeiter, ${ }^{1}$ Patrice Decker, ${ }^{2}$ Christian Jorgensen, 'Marie-Christophe Boissier, 2Pascale Louis-Plence, 'Natacha Bessis. 'EA4222, Li2P, University Paris 13, Sorbonne Paris Cité and Rheumatology Department, Avicenne Hospital, Assistance PubliqueHôpitaux de Paris (AP-HP), Bobigny, 93009 France, Bobigny, France; 2INSERM U844, CHU Saint-Eloi, Université Montpellier 1, CHU Lapeyronie, Montpellier, France

Background and Objectives Studies have demonstrated the clinical efficacy of tocilizumab, a humanised anti-IL-6 receptor (R) antibody $(A b)$, in patients with rheumatoid arthritis (RA). The rational 